Martin Dindoš, Faculty of Mathematics and Physics, Comenius University, Mlynská dolina, 84215 Bratislava, Slovakia. e-mail: dindos@@fmph.uniba.sk

\title{
LIMITS OF TRANSFINITE CONVERGENT SEQUENCES OF DERIVATIVES
}

\begin{abstract}
The paper solves the question whether the limit of transfinite convergent sequence of derivatives is again the derivative. It shows that this problem cannot be solved in the Zermelo-Fraenkel axiomatic system and that this statement is equivalent to the covering number for Lebesgue null ideal being bigger that $\aleph_{1}$. In the second part of the paper author proved an analogue of Preiss's theorem $[\mathrm{P}]$ for the transfinite sequences of derivatives.
\end{abstract}

\section{Introduction}

The convergence of transfinite sequences of functions was introduced in the paper [Sie]. Let $\Omega$ be the first uncountable ordinal number, let $I$ be a real interval and $f_{\xi}: I \rightarrow \mathbb{R}, 1 \leq \xi<\Omega$ be a sequence of real functions. We say that $f: I \rightarrow \mathbb{R}$ is the pointwise limit of this sequence if $f_{\xi}(x) \rightarrow f(x)$ holds for every $x \in I$, i.e.

$$
\forall x \in T \quad \forall \varepsilon>0 \quad \exists \eta<\Omega \quad \forall \xi \geq \eta: \quad\left|f(x)-f_{\xi}(x)\right|<\varepsilon
$$

We shall denote this convergence by $f_{\xi} \rightarrow f$ or more precisely $\lim _{\xi<\Omega} f_{\xi}=f$. An important question is whether the pointwise transfinite convergence preserves some important properties of functions, e.g., continuity or first Baire class. These questions were solved positively in the paper [ک̌ $]$ or [Sie] respectively. In the present paper the question of preserving the property of "being a derivative" will be discussed. The results of this paper can be also used

Key Words: Transfinite sequences, Martin's axiom, Continuum hypothesis.

Mathematical Reviews subject classification: Primary: 40A30 Secondary: 03E50, 46G05

Received by the editors June 17, 1996 
to solve the question of preserving the property of "being an approximately continuous function". This problem was mentioned in the paper $[\check{S}]$ as open.

A partial answer to previous questions gives us the following theorem proved by T. Šalát (oral communication).

Theorem. Let $f_{\xi}: I \rightarrow \mathbb{R}, 1 \leq \xi<\Omega$, be functions differentiable at every point of an interval $I$. Let $f, g: I \rightarrow \mathbb{R}$ be such functions that

$$
f_{\xi} \rightarrow f \text { and } f_{\xi}^{\prime} \rightarrow g \text {. Then } f^{\prime}=g
$$

PROOF. Let $x_{0} \in I$ be an arbitrary point. Then there exists an ordinal number $\eta<\Omega$ such that $f^{\prime}{ }_{\xi}\left(x_{0}\right)=g\left(x_{0}\right)$ holds for every $\eta \leq \xi<\Omega$ : (See $[\mathrm{Sie}])$. It is sufficient to prove that $\frac{f\left(x_{n}\right)-f\left(x_{0}\right)}{x_{n}-x_{0}} \rightarrow g\left(x_{0}\right)$ holds for every sequence $x_{n} \rightarrow x_{0}, n \in \mathbb{N} ; x_{n} \in I \backslash\left\{x_{0}\right\}$.

Since $f_{\xi} \rightarrow f$, there exists an ordinal number $\xi_{0}<\Omega$ such that for all $\xi \geq \xi_{0}$ we have $f_{\xi}\left(x_{k}\right)=f\left(x_{k}\right) \quad(k=0,1,2,3, \ldots)$. But then for any ordinal number $\xi \geq \max \left\{\xi_{0}, \eta\right\}$ we have

$$
\frac{f\left(x_{n}\right)-f\left(x_{0}\right)}{x_{n}-x_{0}}=\frac{f_{\xi}\left(x_{n}\right)-f_{\xi}\left(x_{0}\right)}{x_{n}-x_{0}} \rightarrow{f^{\prime}}_{\xi}\left(x_{0}\right)=g\left(x_{0}\right) \text { for } n \rightarrow \infty .
$$

Let $\Delta$ denote the set of all derivatives on the interval $I$; i.e., all functions $f: I \rightarrow \mathbb{R}$ having primitive functions $F: I \rightarrow \mathbb{R}$ such that $f(x)=F^{\prime}(x)$ for each $x \in I$. Let us introduce the following notation.

$(\mathcal{C H})$ Continuum hypothesis: $\aleph_{1}=2^{\aleph_{0}}$.

$(\mathcal{M A})_{\aleph_{1}}$ Martin's axiom: For a nonempty poset (partially ordered set) $P$ having the $(\mathrm{CCC})^{1}$ property and a family $\left\{D_{j} ; j \in J\right\}$ of dense ${ }^{2}$ sets in $P(\operatorname{card}(J) \leq \kappa)$ there exists a subnet ${ }^{3} Q \subset P$ such that $Q \cap D_{j} \neq \emptyset$. (See [Sch]). We shall use this axiom for $\kappa=\aleph_{1}$.

$(\mathcal{A D D})_{\aleph_{1}}$ The union of $\aleph_{1}$ null sets (Lebesgue measure on $\mathbb{R}$ ) has (Lebesgue) measure zero. This statement can be written as $\operatorname{add}(L)>\aleph_{1}$ where $\operatorname{add}(L)$ is the usual notation for the smallest cardinal $\kappa$ with the property that there are $\kappa$ null sets such that their union is not null.

\footnotetext{
${ }^{1}$ Poset $(P, \prec)$, briefly $P$ has the (CCC) property if every set $Q \subset P$ whose elements are pairwise incompatible is at most denumerable. Two elements $p, q(p \neq q)$ of an poset $P$ are incompatible if there does not exist any element $r \in P$ such that $p \prec r$ and $q \prec r$.

${ }^{2}$ Set $D$ is dense in the poset $P$ if for an arbitrary $p \in P$ there exists $d \in D$ such that $p \prec d$.

${ }^{3} Q$ is subnet of $P$ if $Q \subset P$ and $Q$ is a net; i.e. for every elements $p, q \in Q$ there is an element $r \in Q$ such that $p \prec r$ and $q \prec r$.
} 
$(\mathrm{COV})_{\aleph_{1}}$ There are $\aleph_{1}$ null sets (Lebesgue measure on $\mathbb{R}$ ) covering $\mathbb{R}$. This statement can be written as $\operatorname{cov}(L)=\aleph_{1}$ where $\operatorname{cov}(L)$ is the usual notation for the smallest cardinal $\kappa$ such that the real line is the union of $\kappa$ null sets.

(D) If $f_{\xi}: I \rightarrow \mathbb{R}, \quad 1 \leq \xi<\Omega$ is an arbitrary transfinite pointwise convergent sequence of derivatives, then the limit function $f=$ $\lim _{\xi<\Omega} f_{\xi}$ is also a derivative; i.e. $f \in \Delta$.

$(\mathcal{A C})$ If $f_{\xi}: I \rightarrow \mathbb{R} ; 1 \leq \xi<\Omega$ is an arbitrary transfinite pointwise convergent sequence of approximately continuous functions, then the limit function $f=\lim _{\xi<\Omega} f_{\xi}$ is also approximately continuous.

$(\mathcal{Z F C})$ Zermelo-Fraenkel set theory including the axiom of choice.

Both the continuum hypothesis $(\mathcal{C H})$ and Martin's axiom $(\mathcal{M A})_{\aleph_{1}}$ are statements that are independent with respect to Zermelo-Fraenkel set theory $(\mathcal{Z F C})$ and can be added as a new axiom (of course not both together). The following relations between previous statements were proved in paper [Sch] or they can be easily derived.

$$
\begin{aligned}
(\mathcal{Z F C})+(\mathcal{M A})_{\aleph_{1}} & \Longrightarrow(\mathcal{Z F C})+(\mathcal{A D D})_{\aleph_{1}} \Longrightarrow(\mathcal{Z F C})+\neg(\mathcal{C O V})_{\aleph_{1}} \\
(\mathcal{Z F C})+(\mathcal{C H}) & \Longrightarrow(\mathcal{Z F C})+(\mathcal{C O V})_{\aleph_{1}}
\end{aligned}
$$

The main aim of this paper is to prove following implications.

$$
\begin{aligned}
(\mathcal{Z F C})+\neg(\mathcal{C O V})_{\aleph_{1}} & \Longrightarrow(\mathcal{Z F C})+(\mathcal{D}) \\
(\mathcal{Z F C})+(\mathcal{C O V})_{\aleph_{1}} & \Longrightarrow(\mathcal{Z F C})+\neg(\mathcal{D})
\end{aligned}
$$

which means that $(\mathcal{D})$ and $\neg(\mathcal{D})$ are statements that cannot be derived from $(\mathcal{Z F C})$ because both $(\mathcal{Z F C})+(\mathcal{D})$ and $(\mathcal{Z F C})+\neg(\mathcal{D})$ remain consistent if $(\mathcal{Z F C})$ is consistent. In addition following axiomatic systems are equivalent.

$$
\begin{aligned}
(\mathcal{Z F C})+\neg(\mathcal{C O V})_{\aleph_{1}} & \Longleftrightarrow(\mathcal{Z F \mathcal { C } )}+(\mathcal{D}) \\
(\mathcal{Z F C})+(\mathcal{C O V})_{\aleph_{1}} & \Longleftrightarrow(\mathcal{Z F C})+\neg(\mathcal{D})
\end{aligned}
$$

Remark 1. We also prove that the statements $(\mathcal{A C})$ and $\neg(\mathcal{A C})$ are independent with respect to $(\mathcal{Z F C})$ axioms because from results of this paper the following equivalences can also be derived.

$$
\begin{aligned}
(\mathcal{Z F C})+\neg(\mathcal{C O V})_{\aleph_{1}} & \Longleftrightarrow(\mathcal{Z F \mathcal { C } )}+(\mathcal{A C}) \\
(\mathcal{Z F C})+(\mathcal{C O V})_{\aleph_{1}} & \Longleftrightarrow(\mathcal{Z F C})+\neg(\mathcal{A C})
\end{aligned}
$$




\section{Limits of Pointwise Convergent Transfinite Sequences of Functions When $\neg(\mathrm{CO} \mathcal{V})_{\aleph_{1}}$ Holds.}

In what follows we shall suppose that $\neg(\mathcal{C O V})_{\aleph_{1}}$ or a stronger assumption $(\mathcal{A D D})_{\aleph_{1}}$ holds. First we introduce an auxiliary lemma which we need for the proof of the following Theorem 2 .

Lemma 1 Suppose $\neg(\mathcal{C O V})_{\aleph_{1}}$. Then the inner Lebesgue measure of a union of $\aleph_{1}$ null sets is zero.

Proof. Suppose that this statement does not hold. Then there exist sets $A_{j}$ with $\lambda\left(A_{j}\right)=0, j \in J$ and $\operatorname{card}(J)=\aleph_{1}$ such that

$$
\lambda_{*}(A)>0 \text { where } A=\bigcup_{j \in J} A_{j}
$$

( $\lambda_{*}$ means the inner Lebesgue measure). Then according to a well-known fact $(\mathcal{C O V})_{\aleph_{1}}$ holds; i.e. there exist $\aleph_{1}$ null sets which cover the entire real line which is contrary to the assumption $\neg(\mathcal{C O V})_{\aleph_{1}}$. These sets can be chosen as

$$
B_{j}=\left(C \cup \bigcup_{q \in Q}\left(A_{j}+q\right)\right) \text { for } j \in J \text { where } C=\mathbb{R} \backslash \bigcup_{q \in Q}(A+q) \text {. }
$$

Theorem 2 Let $f_{\xi}: I \rightarrow \mathbb{R}, 1 \leq \xi<\Omega$, be a pointwise convergent transfinite sequence of measurable functions. Let $f=\lim _{\xi<\Omega} f_{\xi}$. Let $(i)$ or $(i i)$ hold.

(i) Axiom $(\mathcal{A D D D})_{\aleph_{1}}$ holds.

(ii) The function $f$ is measurable and axiom $\neg(\mathcal{C O V})_{\aleph_{1}}$ holds.

Then the function $f$ is measurable and there exists an ordinal number $\eta<\Omega$ such that for every $\eta \leq \xi<\Omega f_{\xi}(x)=f(x)$ holds almost everywhere on $I$.

ProOf. We will prove $(i) \Longrightarrow(i i)$. It is sufficient to prove that function $f$ is measurable, because $(\mathcal{A D D})_{\aleph_{1}} \Longrightarrow \neg(\mathcal{C O V})_{\aleph_{1}}$.

In paper [Sch] it was demonstrated that the union and the intersection of at most $\aleph_{1}$ Lebesgue measurable sets is a measurable set provided $(i)$ holds. This fact will be used now.

Since for every $x \in I f_{\xi}(x) \rightarrow f(x)$, there exist an ordinal number $\eta_{x}<\Omega$ such that $f_{\xi}(x)=f(x)$ for every $\eta_{x} \leq \xi<\Omega$. Obviously we have

$$
\{x \in I ; f(x)>\alpha\}=\bigcup_{\eta<\Omega} \bigcap_{\eta<\xi}\left\{x \in I ; f_{\xi}(x)>\alpha\right\}
$$


and therefore the function $f$ is measurable.

Now let (ii) hold. For every ordinal number $\xi<\Omega$ we define

$$
E_{\xi}=\left\{x \in I ; f_{\eta}(x)=f(x) \quad \forall \eta \geq \xi\right\}
$$

The union of $E_{\xi}$ is the interval $I$ and $E_{\xi} \subset E_{\zeta}$ whenever $\xi \leq \zeta$. Without loss of generality we can suppose that $I$ is a bounded interval. Let $c=\sup _{\xi<\Omega} \lambda^{*}\left(E_{\xi}\right)$, where $\lambda^{*}$ is Lebesgue outer measure on I. According to the definition of supremum for every $n \in \mathbb{N}$ there exist an ordinal number $\xi_{n}$ such that

$$
\lambda^{*}\left(E_{\xi_{n}}\right) \geq c-\frac{1}{n}
$$

There exists an ordinal number $\eta<\Omega$ such that $\xi_{n} \leq \eta$ for every $n \in \mathbb{N}$. Therefore for every $n \in \mathbb{N}$ we have

$$
c \geq \lambda^{*}\left(E_{\eta}\right) \geq \lambda^{*}\left(E_{\xi_{n}}\right) \geq c-\frac{1}{n}
$$

Hence $\lambda^{*}\left(E_{\zeta}\right)=c$ for all $\eta \leq \zeta<\Omega$. Let $G$ be a measurable set such that $E_{\eta} \subset G \subset I$ and $\lambda^{*}\left(E_{\eta}\right)=\lambda(G)$.

We want to prove $\lambda(I \backslash G)=0$. Suppose not. We can write

$$
I \backslash G=I \cap G^{c}=G^{c} \cap \bigcup_{\eta \leq \zeta} E_{\zeta}=\bigcup_{\eta<\zeta}\left(E_{\zeta} \backslash G\right) .
$$

If $\lambda(I \backslash G)=\lambda_{*}(I \backslash G)>0$, then according to Lemma 1 there exist $\eta<\zeta$ such that $\lambda^{*}\left(E_{\zeta} \backslash G\right)>0$. The set $G$ is measurable and therefore according to Caratheodory's definition of measurability

$$
\lambda^{*}\left(E_{\zeta}\right) \geq \lambda^{*}\left(E_{\zeta} \backslash G\right)+\lambda^{*}\left(E_{\zeta} \cap G\right)>\lambda^{*}\left(E_{\zeta} \cap G\right) \geq \lambda^{*}\left(E_{\eta}\right)=c
$$

contrary to $\lambda^{*}\left(E_{\zeta}\right)>c$. Therefore $\lambda(I \backslash G)=0$ i.e. $\lambda\left(E_{\eta}\right)=\lambda(I)$. Hence $f$ and $f_{\zeta}$ for fixed $\zeta \geq \eta$ are two measurable functions which disagree on a set of inner measure zero $\left(\left\{x \in I ; f(x) \neq f_{\zeta}(x)\right\} \subset I \backslash E_{\zeta}\right)$ and therefore $f_{\xi}(x)=f(x)$ holds almost everywhere on $I$.

Remark 2. The previous proof shows that the assumption $(\mathcal{A D D})_{\aleph_{1}}$ guarantees measurability of a transfinite limit of measurable functions. The converse of this statement is also true; i.e. the assumption that every transfinite limit of measurable functions is measurable give us that $(\mathcal{A D D})_{\aleph_{1}}$ holds.

Proof. Assume that $\neg(\mathcal{A D D})_{\aleph_{1}}$ holds. Then there exist sets $A_{\xi}$ with $\lambda\left(A_{\xi}\right)=0, \xi<\Omega$ such that

$$
\lambda^{*}(A)>0 \text { where } A=\bigcup_{\xi<\Omega} A_{\xi} .
$$


( $\lambda^{*}$ means the outer Lebesgue measure.) If the set $A$ is non-measurable, we define $B_{\xi}=A_{\xi}, \xi<\Omega$. Otherwise let $B$ be a non-measurable subset of $A$ and define $B_{\xi}=B \cap A_{\xi}, \xi<\Omega$. Then the sets $B_{\xi}$ have measure zero and their union is a non-measurable set. Define

$$
f_{\xi}=\chi \bigcup_{\zeta<\xi} B_{\zeta}
$$

(where $\chi_{C}$ means the characteristic function of the set $C$ ). Then $f_{\xi} \rightarrow \chi_{B}$; i.e. $\chi_{B}$ is a non-measurable function which is the transfinite limit of measurable functions. That is a contradiction and therefore the assumption $\neg(\mathcal{A D D})_{\aleph_{1}}$ cannot hold.

The main theorem of this section is the following.

Theorem $3(\mathcal{Z F C})+\neg(\mathcal{C O V})_{\aleph_{1}} \Longrightarrow(\mathcal{Z F C})+(\mathcal{D})$

(In fact we prove that every pointwise convergent transfinite sequence of derivatives $\left(f_{\xi}\right)_{\xi<\Omega}$ is eventually constant; i.e. there exists an ordinal number $\eta<\Omega$ such that for every $\eta \leq \xi<\Omega f_{\xi}=f \eta$.)

Proof. Let $f_{\xi}: I \rightarrow \mathbb{R} ; 1 \leq \xi<\Omega$ be a pointwise convergent transfinite sequence of derivatives $\left(f_{\xi} \in \Delta\right)$. The function $f=\lim _{\xi<\Omega} f_{\xi}$ is Baire 1 and hence measurable. According to Theorem 2 there exists an ordinal number $\eta<\Omega$ such that for every $\eta \leq \xi<\Omega f_{\xi}(x)=f(x)$ almost everywhere on the interval $I$.

Let $\xi \geq \eta$ be an arbitrary ordinal number. The function

$$
h_{\xi}(x)=f_{\xi}(x)-f_{\eta}(x)
$$

is a derivative and equals zero almost everywhere; so the function $h_{\xi}$ is a Lebesgue integrable derivative. Let $H_{\xi}$ be its primitive function. According to $[\mathrm{R}]$ for Lebesgue integrable derivatives the Newton-Leibnitz formula holds.

$$
H_{\xi}(x)-H_{\xi}(y)=\int_{y}^{x} h_{\xi}(t) d t=0
$$

Hence the function $H_{\xi}$ is constant on the interval $I$; i.e. $h_{\xi}(x)=0$ everywhere. Then for every ordinal number $\xi \geq \eta f_{\xi}=f_{\eta}$, and therefore

$$
f=\lim _{\xi<\Omega} f_{\xi}=f_{\eta} \in \Delta \text {. }
$$

Remark 3. The implication $(\mathcal{Z F C})+\neg(\mathcal{C O V})_{\aleph_{1}} \Longrightarrow(\mathcal{Z F C})+(\mathcal{A C})$ is an easy consequence of Theorem 2, because two approximately continuous functions which agree on a set of full measure have to be equal everywhere. 


\section{Limits of Pointwise Convergent Transfinite Sequences of Functions When $(\mathcal{C O V})_{\aleph_{1}}$ Holds.}

First we introduce a theorem of Petruska and Laczkovich [P-L] that will be used later.

Theorem 4 (Petruska and Laczkovich) Let $H$ be a subset of I. The restriction of each Baire 1 function on $I$ to $H$ can be extended to a derivative on I if and only if $\lambda(H)=0$. This derivative can be chosen bounded if the restriction of the Baire 1 function to $H$ is bounded on $H$.

Remark 4. The analogue of this theorem obtained by replacing the word "derivative" with "approximately continuous function" is also valid.

The main theorem of this section follows.

Theorem 5 Let $(\mathcal{C O V})_{\aleph_{1}}$. The function $f: I \rightarrow \mathbb{R}$ is Baire 1 if and only if there exists a transfinite sequence of derivatives $\left(f_{\xi}\right)_{\xi<\Omega}$ such that $\lim _{\xi<\Omega} f_{\xi}=f$.

Proof. The implication ' $\Longleftarrow$ ' was proved by W. Sierpinski in [Sie]. He showed there that a transfinite limit of Baire 1 functions (i.e. also derivatives) is a Baire 1 function.

We prove the implication ' $\Longrightarrow$ '. Let $f: I \rightarrow \mathbb{R}$ be an arbitrary Baire 1 function. Then there are sets $C_{\xi}, 1 \leq \xi<\Omega$, all of measure zero such that $\mathbb{R}=\bigcup_{\xi<\Omega} C_{\xi}$. Let $D_{\xi}=\bigcup_{\eta \leq \xi} C_{\eta}$. Then sets $D_{\xi}$ have measure zero and $D_{\eta} \subset D_{\xi}$ whenever $\eta \leq \xi$.

According to Theorem 4 there exist derivatives $f_{\xi}$ such that $\left.f\right|_{D_{\xi}}=\left.f_{\xi}\right|_{D_{\xi}}$. Hence Theorem 5 is proved.

Remark 5. This is a stronger version of a theorem published in $[\mathrm{L}]$ where only the implication ' $\Longrightarrow$ ' was proved with the assumption of semi-continuity of function $f$ instead of the assumption of being a Baire 1 function. Theorem 5 gives an affirmative answer to the question asked by the author of [L].

This theorem is also an analogue of Preiss's theorem [P]. He proved that each Baire 2 function is a limit of sequence of derivatives. The assumption $(\mathrm{COV})_{\aleph_{1}}$ provides us a similar theorem for transfinite sequences.

Corollary $6(\mathcal{Z F C})+(\mathcal{C O V})_{\aleph_{1}} \Longrightarrow(\mathcal{Z F C})+\neg(\mathcal{D})$

Proof. Apply Theorem 5 to an arbitrary Baire 1 function which is not derivative. 
Remark 6. Previous proofs can be reformulated to approximately continuous functions instead of derivatives because of Remark 4. Hence also following statement is true.

$$
(\mathcal{Z F C})+(\mathcal{C O V})_{\aleph_{1}} \Longrightarrow(\mathcal{Z F C})+\neg(\mathcal{A C})
$$

\section{References}

[G] Z. Grande, Sur les suites transfinites, Acta Math. Acad. Sci. Hung. no. 30 (1977), 85-90.

[L] J. S. Lipinski, On transfinite sequences of approximately continuous functions Bul. Pol. Acad. Sci. no. 9 (1973), 817-821.

[P] D. Preiss, Limit of derivatives and Darboux-Baire functions, Rev. Roum. Pures et Appl. no. 14 (1969), 1201-1206

[P-L] G. Petruska and M. Laczkovich, Baire 1 functions, approximately continuous functions and derivatives, Acta Math. Acad. Sci. Hung. no. 25 (1974), 189-212.

[R] J. F. Randolph, Basic real and abstract analysis, Academic press Inc., New York and London, 1968.

[Sch] J. K. Shoenfeld, Martin's axiom, Amer. Math. Monthly no. 82 (1975), $610-617$.

[Sie] W. Sierpinski, Sur les suites transfinies convergentes de fonctions de Baire, Fund. Math. no. 1 (1920), 132-141.

[Š ] T. Šalát, On transfinite sequences of B-measurable functions, Fund. Math. (1973), 157-162. 